\title{
A!
}

This is an electronic reprint of the original article.

This reprint may differ from the original in pagination and typographic detail.

Mostafa, M. H.; Dlaz-Rubio, A.; Mirmoosa, M. S.; Tretyakov, S. A.

\section{Multi-Frequency Perfect Absorption by Ultra-Thin Time-Modulated Metasurfaces}

Published in:

2021 15th International Congress on Artificial Materials for Novel Wave Phenomena, Metamaterials 2021

DOI:

10.1109/Metamaterials52332.2021.9577137

Published: 20/09/2021

Document Version

Publisher's PDF, also known as Version of record

Please cite the original version:

Mostafa, M. H., Dlaz-Rubio, A., Mirmoosa, M. S., \& Tretyakov, S. A. (2021). Multi-Frequency Perfect Absorption by Ultra-Thin Time-Modulated Metasurfaces. In 2021 15th International Congress on Artificial Materials for Novel Wave Phenomena, Metamaterials 2021 (pp. 293-295). IEEE.

https://doi.org/10.1109/Metamaterials52332.2021.9577137

This material is protected by copyright and other intellectual property rights, and duplication or sale of all or part of any of the repository collections is not permitted, except that material may be duplicated by you for your research use or educational purposes in electronic or print form. You must obtain permission for any other use. Electronic or print copies may not be offered, whether for sale or otherwise to anyone who is not an authorised user. 


\title{
Multi-Frequency Perfect Absorption by Ultra-Thin Time-Modulated Metasurfaces
}

\author{
M. H. Mostafa, A. Díaz-Rubio, M. S. Mirmoosa, and S. A. Tretyakov \\ Department of Electronics and Nanoengineering, Aalto University, Maarintie 8, 02150, Espoo, Finland \\ mohamed.mostafa@aalto.fi
}

\begin{abstract}
Here, we study the interference phenomena produced in time-modulated metasurfaces when they are illuminated by multiple harmonics opening a new paradigm of coherent time-modulated metasurfaces. In particular, we obtain perfect absorption at multiple frequencies by inducing slow time modulation of a resistive sheet. Also, it will be shown that it is possible to fully tune the absorption by properly designing the modulation parameters.
\end{abstract}

\section{INTRODUCTION}

The design of electrically thin absorbers is an active research field with practical applications, such as communication systems, solar cells, stealth technologies, and optical sensors. The main target has been to design more compact structures and increase the frequency range of full absorption [1].

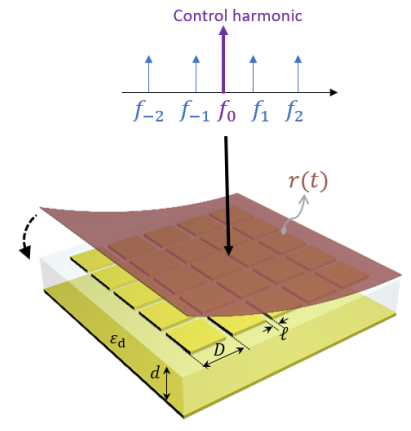

(a)

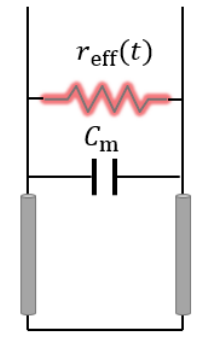

(b)

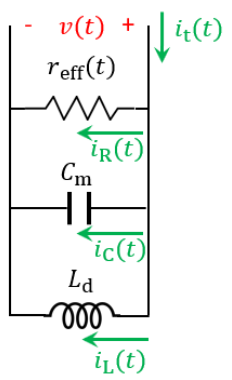

(c)

Fig. 1: (a) Schematic representation of a time-varying reflective metasurface. (b) Equivalent circuit model of the proposed structure for normal incidence. (c) Simplified version of the equivalent model and current distribution.

Recently, a new type of ultrathin absorbers has been proposed based on the coherent illumination of two sides using a splitter or an independent phase-locked generator [2-4]. The concept of coherent metasurfaces have been recently extended to surface-inhomogeneous coherent metasurfaces capable of locally controlling the interference of the illuminating waves along the surface [5]. Here, we go one step further and explore the possibilities offered by slow and coherent time modulation of the metasurface. This approach allows engineering the interchange of energy between several harmonics of incident illumination, boosting the possibilities of perfect absorption. For example, as we will show in this abstract, one can obtain perfect absorption at different frequencies in ultrathin structures by slowly modulating the resistance of the absorbing sheet in time. Additionally, we will evaluate a possibility to dynamically control the level of absorption, for realization of tunable multi-frequency absorbers.

\section{COHEREnT ABSORPTION IN TIME-MOdUlated METASURFACES}

To understand the possibilities offered by coherent illumination in time-modulated surfaces, let us consider the metasurface structure shown in Fig. 1(a). This reflective metasurface consists of a grounded dielectric with the relative permittivity $\varepsilon_{\mathrm{d}}$ and thickness $d$. A periodic array of thin metal patches (electrically small period) is positioned 
on top of the substrate and, over the patches, a time-varying resistive sheet is placed. This metasurface can be modelled by the equivalent circuit in Fig. 1(b). The grounded substrate is represented by a shorted transmission line with the characteristic impedance $\left.\eta_{\mathrm{d}}=\sqrt{\mu_{0} /\left(\varepsilon_{0} \varepsilon_{\mathrm{d}}\right.}\right)$. Considering normal incidence, the shorted transmission line can be viewed as a reactance whose impedance equals $Z_{\mathrm{d}}=j \eta_{\mathrm{d}} \tan \left(k_{\mathrm{d}} d\right)$, where $k_{\mathrm{d}}=\omega \sqrt{\mu_{0} \varepsilon_{0} \varepsilon_{\mathrm{d}}}$. The patch array has a capacitive behaviour and it can be modelled by the sheet capacitance $C_{\mathrm{m}}=2 \epsilon_{\mathrm{eff}} \varepsilon_{0} D \ln \left(\csc \frac{\pi}{2(p+1)}\right) / \pi$ where $\epsilon_{\mathrm{eff}}=\left(\epsilon_{\mathrm{d}}+1\right) / 2$ and $p=(D-\ell) / \ell$. Finally, with the presence of the patch array, the resistive layer is partially shorted and has an effective resistance $r_{\text {eff }}(t)=r(t) \ell /(D-\ell)$ [6].

The metasurface is illuminated by a periodical input signal defined by $v_{\text {in }}(t)=\sum_{n=-N}^{N} a_{n} \cos \left(\omega_{n} t\right)$ where $2 N+1$ is the number of input harmonics. We consider spectra formed by several harmonics symmetrically located at the two sides of the central harmonic $a_{0}=a_{\mathrm{c}}$, whose frequency satisfies $\omega_{0}=\left(\omega_{n}+\omega_{-n}\right) / 2$. Below we will show that this harmonic can be used as a control wave for tuning absorption at other harmonics. In this paper, we will analyze the response of the structure when the effective conductance of the lossy layer varies according to $g_{\mathrm{eff}}(t)=1 / r_{\mathrm{eff}}(t)=g_{0}+\sum_{k=1}^{N} g_{0} m_{k} \cos \left(\omega_{\mathrm{m} k} t+\phi_{\mathrm{m} k}\right)$ where $m_{k}$ are the modulation depths and $\omega_{\mathrm{m} k}=\left|\omega_{n}-\omega_{0}\right|$ are the frequencies of the modulating harmonics. We show that by properly tuning such modulation, perfect absorption at all input frequencies can be achieved.

Let us assume that there is no reflection at all incident frequencies. In this case, the current in the resistive sheet can be written in terms of the time-varying effective conductance as

$$
i_{\mathrm{R}}(t)=[\overbrace{\sum_{n=-N}^{N} a_{n} g_{0} \cos \left(\omega_{n} t\right)}^{T_{1}}]+\overbrace{\left[\sum_{n=-N}^{N} a_{n} \cos \left(\omega_{n} t\right) \sum_{k=1}^{N} g_{0} m_{k} \cos \left(\omega_{\mathrm{m} k} t+\phi_{\mathrm{m} k}\right)\right.}^{T_{2}}] .
$$

Here, $T_{1}$ is the current produced in the resistive element in case of no modulation applied, and $T_{2}$ is the current produced due to modulation. Notice that this expression is only valid when the amplitude of the spurious harmonics is small, otherwise additional harmonics can be created and should be taken into account in the derivations. To meet this condition, $m_{k}$ has to be relatively small. Then, we need to calculate other currents flowing in the system to analyze the response. If we consider the grounded substrate to be electrically thin, we get $Z_{\mathrm{d}} \approx j \omega L_{\mathrm{d}}$ where $L_{\mathrm{d}}=\mu_{0} d$. The current in the inductor and the capacitive layer generated by the metallic patches can be written as

$$
i_{\mathrm{L}}(t)+i_{\mathrm{C}}(t)=\sum_{n=-N}^{N}\left[\frac{1}{L_{\mathrm{d}} \omega_{n}}-C_{\mathrm{m}} \omega_{n}\right] a_{n} \sin \left(\omega_{n} t\right) .
$$

Then the total current reads $i_{t}(t)=T_{1}+T_{2}+i_{\mathrm{L}}(t)+i_{\mathrm{C}}(t)$. Assuming that $g_{0}=1 / \eta_{0}$ and spurious harmonics are negligible, the condition for total absorption is met when $i_{\mathrm{L}}(t)+i_{\mathrm{C}}(t)=-T_{2}$, which is satisfied when we set $\phi_{\mathrm{m} k}=\frac{3 \pi}{2}$ and $\left|0.5 a_{\mathrm{c}} g_{0} m_{k}\right|=\left|\left[\frac{1}{L_{\mathrm{d}} \omega_{n}}-C_{\mathrm{m}} \omega_{n}\right] a_{n}\right|$. As a result, perfect absorption at multiple frequencies of a given spectrum can be obtained by engineering the modulation parameters. Note that there is no limit for improving the absorption at side bands if one can increase the amplitude of the central harmonic $a_{\mathrm{c}}$. Importantly, these results are obtained assuming slow modulation, where the modulation frequency is much smaller than the input frequencies, which is more practical than the double frequency modulation usually used in parametric devices.

\section{Results}

To validate the results obtained above, we consider an input signal with four harmonics at $0.95 \mathrm{GHz}, 0.97$ $\mathrm{GHz}, 1.03 \mathrm{GHz}$, and $1.05 \mathrm{GHz}$, while the central harmonic is at $1 \mathrm{GHz}$. Those harmonics give rise to modulation frequencies $30 \mathrm{MHz}$ and $50 \mathrm{MHz}$. The array of patches is designed to produce a resonance with the shorted transmission line at $1 \mathrm{GHz}$ with $L_{\mathrm{d}}=3 \mathrm{nH}, C_{\mathrm{m}}=8.44 \mathrm{pF}$, and $g_{0}=1 / \eta_{0}$. For simplicity, we assume $a_{ \pm 2}=$ $a_{ \pm 1}=1$. Perfect absorption of all harmonics is realized with $\phi_{\mathrm{m} 1,2}=3 \pi / 2, a_{\mathrm{c}}=10, m_{1}=0.245$, and $m_{2}=0.38$. Note that we can decrease $m_{k}$ even more by increasing $a_{c}$.

Now, we will discuss numerical results obtained from SIMULINK. Figure 2(a) shows the time modulated effective resistance, and the absorption spectrum is shown in Fig. 2(b) (bottom panel). In the absence of modulation, the metasurface provides narrowband absorption, however, when modulation is present, absorption is boosted nearly to perfection for all harmonics. For example, at $0.95 \mathrm{GHz}$, we increase the absorption from less than $50 \%$ to $99 \%$. 
The reflected voltage amplitude is shown in the top panel where we can see that the reflected harmonics have negligibly small amplitudes compared to the input signal amplitudes $\left(a_{ \pm 2}=a_{ \pm 1}=1\right)$. As expected, due to the small amplitude of the modulation function, the amplitude of the high-order harmonics is small, validating the model assumption. In addition to boosting absorption to the maximum, it is also possible to tune the absorption to any value by varying $a_{c}$. Figure 2(c) shows the absorption as a function of $a_{c}$. Circular symbols are for $\phi_{\mathrm{m} 1,2}=3 \pi / 2$ where absorption is enhanced, while star symbols are for $\phi_{\mathrm{m} 1,2}=\pi / 2$ where absorption is reduced. It is important to notice that every harmonic can be controlled individually by assigning different values for $\phi_{\mathrm{m} 1}$ and $\phi_{\mathrm{m} 2}$. As a result, the absorption becomes fully tunable by properly engineering the control and modulation parameters. Notice that the modulation does not reduce the absorption at the central frequency as the terms expressing coupling to this frequency cancel out due to the symmetry. Finally, it is important to note that the metasurface does not pump energy to the fields, meaning that the total energy scattered by the metasurface is smaller than the illumination energy. In practice, this ensures that the energy used to generate and maintain the modulation will not be transferred to the scattered fields. In general, the amount of energy consumed to modulate the resistive layer depends on the actual implementation being impossible to have an estimation at this stage of the work. During our talk, we will extend these results and present a comprehensive study of the absorption properties of time-varying resistive sheets and their applications to different types of metasurfaces.

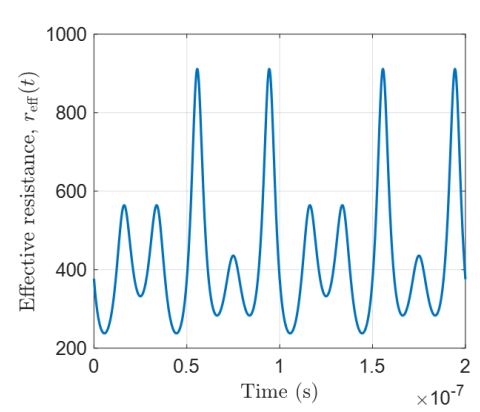

(a)

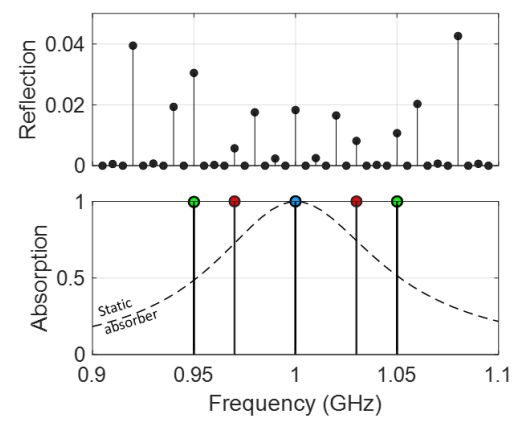

(b)

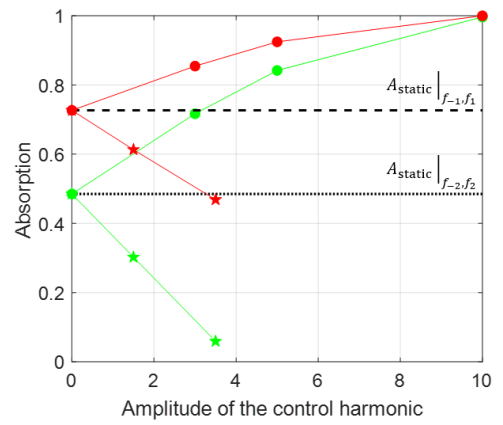

(c)

Fig. 2: Simulation at $f_{0}=1 \mathrm{GHz}$ when $C_{\mathrm{m}}=8.44 \mathrm{pF}$ and $L_{\mathrm{d}}=3 \mathrm{nH}$. As a practical example, $\varepsilon_{d}=12$, $D=\lambda_{0} / 10=30 \mathrm{~mm}, g=298 \mu \mathrm{m}$, and $d=2.38 \mathrm{~mm} \approx \lambda / 125$. (a) The effective resistance required to obtain perfect absorption at $0.95 \mathrm{GHz}, 0.97 \mathrm{GHz}, 1 \mathrm{GHz}, 1.03 \mathrm{GHz}$, and $1.05 \mathrm{GHz}$ when $a_{\mathrm{c}}=10$ and $a_{ \pm 2}=a_{ \pm 1}=1$. (b) Amplitude of the reflected harmonic (top panel) and absorption spectra (bottom panel). (c) Tunable absorption by controlling the amplitude of the central harmonic and the phase of the modulating signal. Values with circular symbols are obtained with a modulation phase $\phi_{\mathrm{m} 1,2}=\frac{3 \pi}{2}$ and star symbols are for modulation phase $\phi_{\mathrm{m} 1,2}=\frac{\pi}{2}$.

ACKNowledgement: This work was supported by the Academy of Finland under the grants 330957 and 330260. Also, the authors thank P. Jayathurathnage, G. Ptitcyn, X. Wang, and N. Ha-Van for having useful discussions.

\section{REFERENCES}

[1] Y. Ra'di, C. Simovski, and S. Tretyakov, Thin perfect absorbers for electromagnetic waves: Theory, design, and realizations, Phys. Rev. Appl, vol. 3, no. 3, 037001, 2015.

[2] D. G. Baranov, A. Krasnok, T. Shegai, A. Alu, and Y. Chong, Coherent perfect absorbers: linear control oflight with light, Nat. Rev. Mater., vol. 2, 170642017.

[3] S. Li, J. Luo, S. Anwar, S. Li, W. Lu, Z. H. Hang, Y. Lai, B. Hou, M. Shen, and C. Wang, Broadband perfect ab-sorption of ultrathin conductive films with coherent illu-mination: Superabsorption of microwave radiation, Physical Review B, vol. 91, 220301, 2015.

[4] X. Fang, K. F. MacDonald, and N. I. Zheludev, Controlling light with light using coherent metadevices: all-optical transistor, summator and invertor, Light Sci.Appl., vol. 4, e292, 2015.

[5] F. Cuesta, G. Ptitcyn, M. Mirmoosa, and S. Tretyakov, Coherent Retroreflective Metasurfaces, arXiv preprint arXiv:2012.14391, 2020.

[6] W. Wang and S. Tretyakov, Toward ultimate control of terahertz wave absorption in graphene, IEEE Transactions on Antennas and Propagation, vol. 67, no. 11, pp. 2452-2461, 2019. 